\title{
Lossless Image Compression Using A Simplified MED Algorithm with Integer Wavelet Transform
}

\author{
Mohamed M. Fouad \\ Department of Computer Engineering, Military Technical College \\ Kobry Elkoppa, Cairo, Egypt \\ mmafoad@ieee.org \\ Richard M. Dansereau \\ Department of Systems \& Computer Engineering, Carleton University \\ Ottawa, Ontario, Canada \\ rdanse@sce.carleton.ca
}

\begin{abstract}
In this paper, we propose a lossless (LS) image compression technique combining a prediction step with the integer wavelet transform. The prediction step proposed in this technique is a simplified version of the median edge detector algorithm used with JPEG-LS. First, the image is transformed using the prediction step and a difference image is obtained. The difference image goes through an integer wavelet transform and the transform coefficients are used in the lossless codeword assignment. The algorith $\mathrm{m}$ is simple and test results show that it yields higher compression ratios than competing techniques. Computational cost is also kept close to competing techniques.
\end{abstract}

Index Terms - Lossless, image compression, median edge detector, integer wavelet transform, Joint Photographic Experts Group.

\section{INTRODUCTION}

Image compression is important when it comes to storing or transmitting images. The idea behind image compression is to represent the image in the smallest number of bits while maintaining the essential information of the image. The most popular image compression techniques perform compression where both information redundancy and perceptual redundancy are removed with minimal image quality degradation. However, some applications require lossless reconstruction like for some medical image or satellite image applications where legal or technical requirements oblige that no bits have changed.

Lossless compression algorithms were developed to meet both the need of compression and the requirement for no change in image bits for certain applications. In the lossless group of image compression algorithms, the desire is to reduce the number of bits to represent the image in a way that is reversible without any data loss. Many algorithms have been developed to provide lossless compression, such as lossless Joint Photographic Experts Group (JPEG-LS) and CALIC, but all provide a small compression ratio compared with lossy compression. The low ratio of lossless compression algorithms is expected since only information redundancy is removed while perceptual redundancy is left unaltered [1].

In [2], the authors presented several low complexity lossless and lossy compression approaches using the step size matrix with preserving the decoded image data unchangeable. In [3], the authors showed a method of encoding color images in a visually lossless manner using JPEG2000 by measuring some visibility thresholds used for quantization of subband signals in JPEG2000. Those thresholds are experimentally obtained from statistically modeled quantization distortion that is based on the distribution of wavelet coefficients and the dead-zone quantizer of JPEG2000. The resulting thresholds are then adjusted for locally changing backgrounds through a visual masking model to be included in the final code stream for visually lossless quality under certain viewing conditions.

In [4], the authors presented lossless and near-lossless compression algorithms for multichannel signals based on image and volumetric coding. Those multichannel signals not only have significant correlation among spatially adjacent channels, but also they are correlated across time. This correlation is imposed to the compression algorithm yielding a specifiable maximum error between orig inal and reconstructed signals. In [5], the authors presented an integer multi-wavelet transform for lossless image compression. The transform coefficients are coded with magnitude set coding without considering the sign, which make uses of magnitude setvariable length integer representation, with setting the sign information of the coefficients to zero.

In [6], the authors presented a colored image compression algorithm by only storing its corresponding grayscale one and some other elective color pixel seeds with regression models to predict the missing colors at the end. Finally, their algorithm can achieve better compression ratio and better colorization quality by minimizing the total color prediction error. In [7], the authors presented a new concept of robust fractal image compression that is less computational cost using some optimization techniques, such as genetic algorithms, by decreasing the search space for finding the self 
similarities in the given image yielding robustness against outliers in that image.

In [8], the authors presented an image compression assuming that the bit data contaminated by noise can be manipulated without affecting image quality. Their compression method requires first estimating the noise level for each pixel, then, identifying those bits contaminated by noise and setting them to zero, and finally, performing a lossless data compression on the processed image. In [9], the authors showed a lowcomplexity integer-reversible spectral-spatial transform that allows for efficient lossless compression of colorfilter-array images. Their idea relies on mapping the pixel array values into a format that can be directly compressed in a lossless progressive-to-loss less manner by an existing typical image coder, such as JPEG 2000 or JPEG XR.

In [10], the authors proposed a prediction sheme for lossless image compression in which the optimal correlated prediction for block of pixels are simultaneously determined using the least code length. Their idea not only uses the spatial statistical correlation for the optimal prediction directly based on 2-D contexts, but also formulates the data-driven structural interdependencies to make the prediction error coherent with the underlying probability distribution for compression.

In [11], the authors proposed a lossless image compression method using the neighboring pixels to ultimately obtain the reference point values. Their idea relies on scanning every pixel row by row and assigns a 2-bit reference point value to each pixel by comparing its intensity value to the neighboring pixels' intensity values. The compression is achieved as only the information of 2-bit reference point values for all pixels and certain intensity values are required for storage. That lossless compression technique was extended in [12] by associating a threshold value is in the compression process, thus different compression ratios can be achieved by varying the threshold values and lossless compression is performed if the threshold value is set to zero.

In [13], the authors proposed a reversible color transform followed by a grayscale encoder to provide higher coding gain than the independent compression of each channel does. Their idea relies on compressing the luminance channel, $Y$, by a conventional lossless image coder, such as JPEG-LS or JPEG2000 lossless. Then, the chrominance channels, $C_{\mathrm{u}}$ and $C_{\mathrm{v}}$, are compressed with another method as their prediction errors have different statistics from those of luminance channel, $Y$. In [14], the authors presented a lossless image compression algorithm based on adaptive neural networks (ANN). Their idea depends on enhancing the ANN by introducing contexts, variable training, window size, and post-processing.

Since the wavelet transform provides high quality compression in the lossy field, many developers are turning to the wavelet transform for lossless compression, including the JPEG2000 lossless algorithm. For lossless compression, the integer-to-integer family of wavelet transform [15] was developed to remove quantization errors that prevent lossless compression with the regular wavelet transform. Other algorithms not only make use of the wavelet transform, but try to combine it with other techniques to increase the compression ratio. However, there is still desire to develop algorithms that can further enhance and increase the compression ratio.

In this paper, we modify the conventional median edge detector (MED) algorithm, used in JPEG-LS [16]. Then, we propose an image compression technique that us es the modified MED in combination with the integer wavelet transform to increase the compression ratio with comparable computational cost. The proposed technique is inspired by Shi and Xie [17] that combines the integer wavelet transform with differential pulse code modulation (DPCM).

Note that the technique shown by Shi and Xie [17] is outperformed by Li and Zhu [18], which uses an integer wavelet packet transform in conjunction with DPCM. In the proposed technique, the image is first transformed using the modified MED algorithm. Then, the result is transformed using the integer wavelet transform. Lossless Huffman coding is then applied to the transformed image to obtain a lossless compressed image that can be stored or transmitted. That compressed image is decompressed, reconstructing the original image by reversing each step in order.

This paper is organized as follows. Section II and Section III give brief backgrounds to the lossless compression concept and the integer wavelet transform, respectively. Section IV presents the approach shown by Shi and Xie [17] due to its closeness to our approach. Section $\mathrm{V}$ presents the proposed compression technique. In Section VI, experimental results are presented using commonly used data set of images. Finally, conclusions are drawn in Section VII.

\section{LOSSLESS COMPRESSION}

A Lossless compression plays an important role in image compression in fields such as medical imaging, where due to information sensitivity and legal requirements no information can be removed from the image once it has been initially digitized. However, compression ratios attained from lossless compression are very s mall, normally revolving around a 2:1 ratio as shown by [17]. The ratio that lossless compression provides is small compared to lossy compression ratios that can yield 300:1. However, great compression ratios would be achieved only when the perceptual redundancy is exploited.

Compression of images normally consists of three steps: transformation, quantization, and codeword assignment. However, with lossless compression, and since quantization introduces quantization errors that prevent perfect reconstruction, lossless compression does not have a quantization step. The quantization step is normally used to turn the transformation coefficients from their float format to an integer format. With lossless compression, and to afford removing quantization, the 
algorithm must make use of a transform that yields only integer coefficients and allows for perfect reconstruction.

Lossless compression algorithms make use of prediction based algorithms that only result in integer values. The prediction algorithm used is known and used by both the encoder and decoder of the image which allows it to be a perfect reconstruction transform. Regardless of the prediction algorithm used, the coefficients used for codeword assignment represent the difference between the predicted pixel value and the actual pixel value of the image. By making good predictions, the error is made small which in turn yields for shorter codeword assignments. Some of the common prediction algorithms used for lossless compression are differential pulse code modulation (DPCM) and the median edge detector (MED) [1] that is used with JPEGLS [19]. The codeword assignment can be done using Huffman codeword, SPIHT, or any arithmetic codeword assignment algorithm, as long as it allows for perfect reconstruction.

\section{INTEGER WAVELET TRANSFORM}

The integer wavelet transform was developed by [15] based on the lifting scheme presented by [17]. Although this transform does not make use of dilation and translation of a mother wavelet like the regular wavelet transform, it is able to keep the multi-resolution properties of the wavelet transform. To obtain the low frequency component $S_{\mathrm{i}-1}$ and the high frequency coefficients $d_{i-1}$ that the wavelet transform forms by transforming a signal $s_{\mathrm{i}}$, the integer wavelet transform operates on three steps: split, prediction, and update.

\section{A. Split Step}

This step is also called the "lazy wavelet" transform [20]. The split operation simply splits the signal $s_{i}$ into even $s_{i-1}$ and odd $s_{i-1}$ subsets, as

$\operatorname{Split}\left(s_{i}\right)=\left(\right.$ even $\left._{i-1} ; \operatorname{odd}_{i-1}\right)=\left(s_{i-1} ; d_{i-1}\right)$.

\section{B. Compression Step}

Due to the high correlation between the odd and even coefficients in an image, the subset $d_{i-1}$ can be predicted efficiently from subset $s_{i-1}$. Once the prediction is made, the signal $s_{i}$ can be replaced by subset $s_{i-1}$ and prediction error between the predicted $d_{i-1}$ and the real values of $d_{i-1}$ obtained from the split.

\section{Update Step}

The update step is performed in order to enhance the subset $s_{i-1}$ after the prediction step. The update step is needed because some of the properties in data set $s_{i-1}$ don't match with these of the original data set. After the three steps are performed on the signal, the result will be low pass coefficients $s_{i-1}$ and high pass coefficients $d_{i-1}$. Figure 1 shows both the analysis and the synthesis operations for the integer wavelet transform.

Different transforms can be obtained by changing the was built based on the Haar wavelet. The Haar integer transform, also known as the $\mathrm{S}$ integer wavelet transform is described by [17] as

$$
\begin{aligned}
& d_{i-1, l}=s_{i, 2 l+1}-s_{i, 2 l} \\
& s_{i-1, l}=s_{i, 2 l+1}+\text { int }\left[d_{i-1, l} / 2\right],
\end{aligned}
$$

Where $s_{\mathrm{i}, 2 \mathrm{l}+1}$ is the odd subset, $s_{\mathrm{i}, 2 \mathrm{l}}$ is the even subset and int[.] denotes the operation of truncating the value into an integer. Other integer wavelet transforms were created by changing the prediction step or performing a second prediction step after the initial steps to enhance the prediction values. These integer wavelet transforms include the $\mathrm{S}+\mathrm{P}$ transform proposed by Said and Pearlman [21], and the $(2,2)$ transform which is the standard used in the JPEG2000 lossless compression option.

\section{RELATED WORK}

The algorithm presented in this paper is inspired by that shown in [17]. Due to the close relation between the techniques, and because the algorithm will be simulated to generate results for comparison with the proposed algorithm, we describe the algorithm of [17] in this section.

What Shi and Xie [17] proposed was to perform the integer wavelet transform, not on the image itself, but on the difference image that is obtained by passing the image through a DPCM transformer. DPCM is an algorithm used for analog to digital conversion and for data transmission where a prediction error is trans mitted instead of the actual value. Both coder and decoder use the same prediction algorithm and the decoder is able to perform a perfect reconstruction on the signal using its prediction and the received prediction error. DPCM can be extended to perform prediction and obtain a difference image by predicting each pixel value from the two previously seen adjacent pixels. The neighborhood of pixels used in the prediction process includes a left-toright, top-to-bottom scan pattern. The predicted pixel is then replaced by the prediction error between the predicted value and the real value in the image. For the first row and first column in the image, no prediction is performed and the pixel values are simply replaced by the difference between the pixel value at that location and the value of the pixel that comes before it. The algorithm is presented below in equation (4). Reconstruction is done by (5).

$$
\begin{aligned}
& \left\{\begin{aligned}
x_{i, j} & =x_{i, j}+\operatorname{int}\left[\operatorname{average}\left(x_{i-1, j} ; x_{i, j-1}\right)\right] \\
x_{i, 1} & =x_{i, 1}+x_{i-1,1} \\
x_{1, j} & =x_{1, j}+x_{1, j-1}
\end{aligned}\right. \\
& \left\{\begin{aligned}
x_{i, 1} & =x_{i, 1}+x_{i-1,1} \\
x_{1, j} & =x_{1, j}+x_{1, j-1} \\
x_{i, j} & =x_{i, j}-\operatorname{int}\left[\operatorname{average}\left(x_{i-1, j} ; x_{i, j-1}\right)\right]
\end{aligned}\right.
\end{aligned}
$$


where $2 \leq i, j \leq M, N$ and ( $i, j)$ is in a (row, column) format with a left-to-right, top-to-bottom scan pattern.

Once the difference image is ready, it is passed through the wavelet transform where the Haar integer transform is used as shown by [17]. The transformed image then goes through a codeword assignment block. Shi and Xie [17] use SPIHT coding for codeword assignment.

\section{PRoposed IMAge COMPRESSION TECHNIQUE}

The compression algorithm proposed in this paper is cast in three steps. First, the image is transformed to a difference image using a prediction algorithm. Second, the difference image is transformed using integer wavelet transform. Finally, the wavelet coefficients are encoded using lossless codeword assignment. The block diagram of the proposed algorithm presented can be seen in Figure 2.

In the proposed technique, a modified version of the Median Edge Detector (MED) algorithm is exploited, instead of DPCM used by Shi and Xie [17]. First, the conventional MED algorithm is shown in the next subsection. Then, the modified version of MED is shown in Section V-B.

\section{A. Median Edge Detector}

This algorithm was first developed in [22] and then presented here in conjunction with the lossless JPEG image compression known as JPEG-LS. MED predicts the pixel value using its previously seen neighbors. MED specifies three neighbors in the prediction: the pixel to the north $N$, the pixel to the west $W$, and the pixel to the northwest $N W$ of the pixel of interest. The prediction neighborhood is shown in Figure 3. The prediction pixel $P[i, j]$ is specified by,

$P[i, j]=\left\{\begin{array}{lc}\min (N, W), & N W>\max (N, W) \\ \max (N, W), & N W<\max (N, W) \\ N+W-N W, & \text { otherwise }\end{array}\right.$

\section{B. Modified Median Edge Detector}

The proposed prediction step is very close to MED discussed in Section V.A. We use the prediction neighborhood shown in Figure 3. (6) can be simplified to median $(N, W, N+W-N W)$. The modification on MED is done to reduce the prediction overhead of MED in calculating the difference between the $N W$ pixel and both $N$ and $W$ pixels.

Also, the prediction modification allows the algorithm to predict the pixel based on the already seen neighbor with the highest probability of occurrence. If the prediction neighborhood has more than one pixel with the same value, then that value is used in the prediction, since it has the highest probability of repeating. If all three values are different, the median value of $(N, W$, $N W$ ), is simply chosen, since it will be either equal to the average value, or close to it.
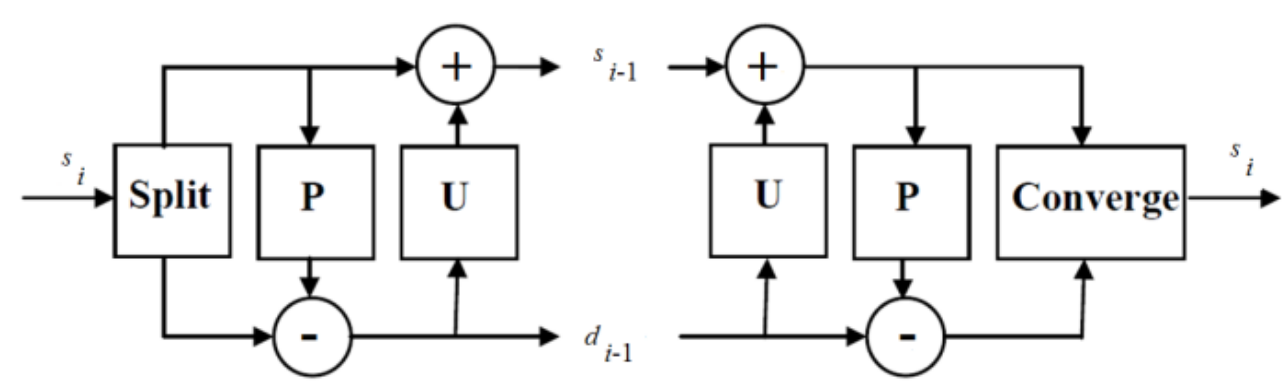

Figure 1. Forward and inverse integer wavelet transform. P and U denote the prediction and update operations, respectively.
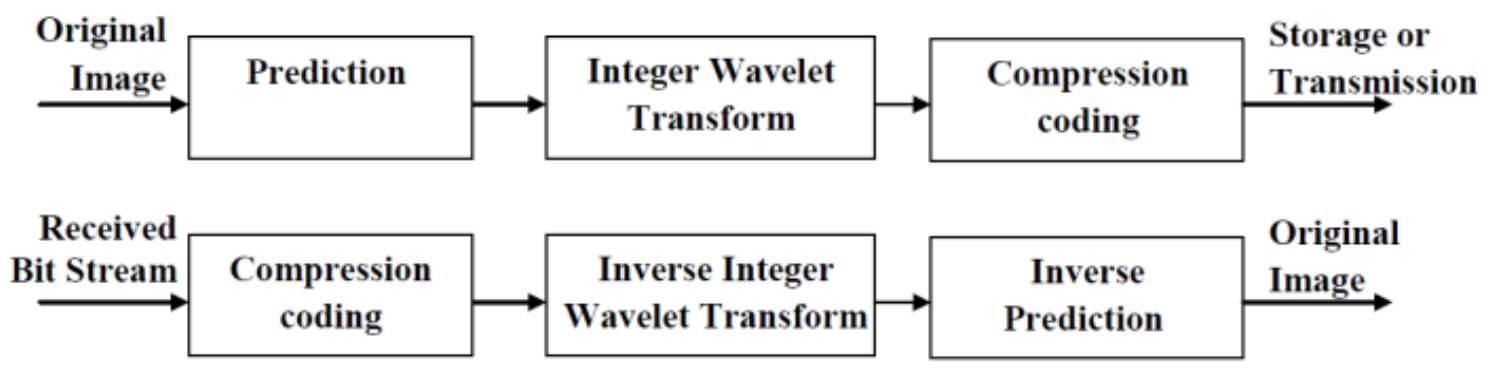

Figure 2. Block diagram of prop osed technique. Top: compression block diagram. Bottom: decompression block diagram. 


\begin{tabular}{|c|c|}
\hline$N W$ & $N$ \\
\hline$W$ & $P[i, j]$ \\
\hline
\end{tabular}

Figure 3. Pixel of interest, $\mathrm{P}$, and its prediction neighborhood

\section{EXPERIMENTS AND RESULTS}

Our implementation runs on a $2.4 \mathrm{GHz}$ Core 2 Duo, with 4 GB of RAM. We compare the proposed compression technique to that of [17]; referred to as DPCM-IWT and that of [18]; referred to as DPCM-IWPT. In the proposed technique, we use the Haar integer transform as shown by [18] in the wavelet transform block shown in Figure 2, for simplicity. Note that 3-level decomposition is used when compression is performed. As well, we use the Huffman coder for the compression coding block shown in Figure 2. Also, we use the commonly used set of images, such as Barbara, Boat, Bridge, Cameraman, Goldhill, Lena, and Peppers.

Table 1 shows the compression ratios and computational cost (sec.) using competing techniques with the data set aforementioned. The results in Table 1 show that the proposed technique outperforms the DPCM-IWT and the DPCM-IWPT techniques by an average improvement of $10.5 \%$ and $5.9 \%$, respectively, in terms of compression ratio. Also, Table 1 illustrates that the proposed technique produces comparable computational cost in seconds. Note that the computational cost denotes the compression time.

TABLE I. Compression Ratio And Computational Cost (SeC.) using i) DPCM-IWT [17], II) DPCM-IWPT [18] AND III) THE PROPOSED TECHNIQUE.

\begin{tabular}{|l|c|c|c|c|c|c|}
\hline \multirow{2}{*}{ Image } & \multicolumn{3}{|c|}{ Image Compression Ratio } & \multicolumn{3}{c|}{ Computational Cost (seconds) } \\
\cline { 2 - 7 } & (I) & (II) & (III) & (I) & (II) & (II) \\
\hline Barbra & 2.57 & 2.64 & 2.70 & 0.31 & 0.27 & 0.26 \\
\hline Boat & 2.52 & 2.75 & 2.96 & 0.29 & 0.26 & 0.25 \\
\hline Bridge & 2.38 & 2.42 & 2.54 & 0.30 & 0.25 & 0.27 \\
\hline Cameraman & 2.35 & 2.48 & 2.59 & 0.28 & 0.24 & 0.26 \\
\hline Goldhill & 2.56 & 2.56 & 2.69 & 0.32 & 0.28 & 0.27 \\
\hline Lena & 2.68 & 2.89 & 3.09 & 0.33 & 0.26 & 0.28 \\
\hline Pepp ers & 2.73 & 2.83 & 3.11 & 0.31 & 0.25 & 0.27 \\
\hline Average & 2.54 & 2.65 & 2.81 & 0.31 & 0.27 & 0.26 \\
\hline
\end{tabular}

\section{CONCLUSIONS}

In this paper, a lossless compression algorithm is proposed combining the integer wavelet transform with a prediction pre-processing step to the image. As well, a prediction step is proposed based on a modification to the MED prediction algorithm used by JPEG-LS. The proposed technique is compared to approaches that combine the differential pulse code modulation with either integer wavelet transform or integer wavelet packet transform. The results shows that the proposed compression technique surpasses competing ones by an average improvement of $10.5 \%$ and $5.9 \%$, respectively, in terms of compression ratio, preserving the computational cost in a comparable manner. However, we believe that there is more room for improvement to achieve even better performance.

\section{ACKNOWLEDGMENT}

We thank anonymous referees for their constructive comments.

\section{REFERENCES}

[1] M. J. Weinberger, G. Seroussi, and G. Sapiro. The LOCO-I lossless image compression algorithm: principles and standardization into JPEG-LS. IEEE Transactions on Image Processing, 2000, 9(8):13091324.

[2] Chu, W.C., On lossless and lossy compression of step size matrices in JPEG coding. International Conference on Computing, Networking and Communications, 2013, 103-107.

[3] H. Oh, A. Bilgin, M. Marcellin. Visually Lossless Encoding for JPEG2000. IEEE Transactions on Image Processing, 2013, 22(1):189-201.

[4] K. Srinivasan, J. Dauwels, M. Reddy. Multichannel EEG Compression: Wavelet-Based Image and Volumetric Coding Approach. IEEE Journal of Biomedical and Health Informatics, 2013, 17(1):113120. 
[5] K. Rajakumar, T. Arivoli. Implementation of Multiwavelet Transform coding for lossless image compression. International Conference on Information Comm. and Embedded Systems, 2013, 634-637.

[6] Chiyuan Zhang, Xiaofei He. Image Compression by Learning to Minimize the Total Error. IEEE Transactions on Circuits and Systems for Video Technology, 2013, 23(4): 565-576.

[7] K. Uma, P. Palanisamy, P. Poornachandran. Comparison of image compression using GA, ACO and PSO techniques. International Conference on Recent Trends in Information Technology, 2011, 815-820.

[8] Tzong-Jer Chen, Keh-Shih Chuang. A pseudo lossless image compression method. The $3^{\text {rd }}$ International Congress on Image and Signal Processing, 2010, 2:610-615.

[9] H. Malvar, G. Sullivan. Progressive-to-Lossless Compression of Color-Filter-Array Images Using Macro-pixel Spectral-Spatial Transformation. Data Compression Conference, 2012, 3-12.

[10] W. Dai, H. Xiong, "Discriminative structured set prediction modeling with max-margin Markov network for optimal lossless image coding," IEEE Proceedings of Visual Communications and Image Processing, 2012, 1-6.

[11] Y.F. Tan, W.N. Tan, K.Y. Tan. Lossless Image Compression Utilizing Reference Points Coding. Proceedings of the $3^{\text {rd }}$ International Conference on Dig ital Image Processing, 2011, 80092-E:1-5.

[12] Yi-Fei Tan, Wooi-Nee Tan. Image compression technique utilizing reference points coding with threshold values. International Conference on Audio, Language and Image Processing, 2012, 74-77.

[13] Seyun Kim, Nam Ik Cho. A lossless color image compression method based on a new reversible color transform. IEEE Proceedings of Visual Communications and Image Processing, 2012, 1-4.

[14] G. Ulacha, R. Stasinski. Imp roving neural network approach to lossless image coding," Picture Coding Symposium, 2012, 173-176.

[15] A. R. Calderbank, I. Daubechies, W. Sweldens, and B.-L. Yeo. Wavelet transforms that map integers to integers. Technical report, Department of Mathematics, Princeton University, U.S.A., 1996.

[16] M. J. Weinberger, G. Seroussi, G. Sapiro. From LOCO-I to the JPEG-LS standard. Proceedings of the IEEE International Conference on Image Processing, 1999, 4:68-72.

[17] Min Shi, Shengli Xie. A lossless image compression algorithm by combining DPCM with integer wavelet transform. Proceedings of the IEEE Circuits and Systems Symposium on Emerging Technologies: Frontiers of Mobile and Wireless Communication, 2004, 293 - 296.

[18] Hua Li, Yiming Zhu. Lossless Image Compression Based on DPCM-IWPT. International conference on Colloquium on Computing, Communication, Control, and Management, 2008, 157-160.
[19] Michael D. Adams. The JPEG-2000 Still Image Compression Standard. Technical report no. N2412, ISO/IEC JTC 1/SC 29/W G, Dept. of Electrical and Computer Engineering, University of Victoria, Canada, 2005.

[20] A. M. Gavrovska, M. P. Paskas, I. S. Reljin. An example of wavelet denoising using lazy transform and the lifting scheme. Proceedings of the Teleco mmunications Forum, 2011, 615-618.

[21] A. Said, W. A. Pearlman. Reversible image compression via multi-resolution representation and predictive coding. SPIE conference on Visual Communications and Image Processing, 1993, 2094: 664-674.

[22] M. M. Fouad, R. M. Dansereau. A lossless image compression technique by combining integer wavelet transform with a simplified MED algorithm. International Conference on Electrical and Computer Systems, 2012, 129-1 - 129-6.

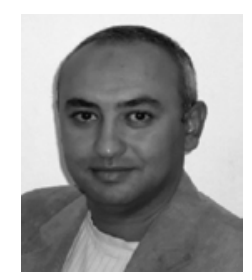

Mohamed M. Fouad, received the Bachelor engineering (honors, with great distinction) and Masters' engineering degrees from the Military Technical College (MTC), Cairo, Egypt, in 1996 and 2001, respectively. As well, he received the Ph.D. degree in Electrical and Computer engineering from Carleton University, Ottawa, Ontario, Canada, in 2010. He is currently a faculty member with the Department of Computer Engineering, MTC. His research interests are in online handwritten recognition, image registration, image reconstruction, super-resolution, video compression and multiview video coding.

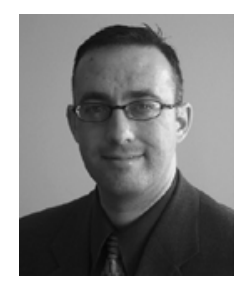

Richard M. Dansereau, received the B.Sc. and Ph.D. degrees in Computer Engineering from the Department of Electrical and Computer Engineering, University of Manitoba, Winnipeg, MB, Canada, in 1995 and 2001, respectively. From 1996 to 2000, he was a researcher with the Telecommunications Research Laboratories (TRLabs) dealing with wavelet and fractal image analysis. From 1999 to 2000, he was also with SpectraWorks, Inc. developing MPEG-DB satellite communication systems. From 2000 to 2001, he was an Instructor and Research Engineer in the Department of Electrical and Computer Engineering at the Georgia Institute of Technology, Atlanta, GA, and was also part of the Center for Signal and Image Processing at Georgia Tech. In September 2001, he joined the Department of Systems and Computer Engineering, Carleton University, ON, Canada, where he is currently an Associate Professor. 